\title{
Miotomía endoscópica por vía oral (POEM) en un paciente pediátrico para tratamiento de la acalasia esofágica
}

\author{
Per oral endoscopic myotomy in a pediatric patient with achalasia \\ Ricardo Mejía ${ }^{\mathrm{a}, \mathrm{b}}$, Josefina Sáez ${ }^{\mathrm{c}}$, Francisco Aranda ${ }^{\mathrm{d}}$, Juan Carlos Pattillo \\ José Fernando Vuletin ${ }^{\mathrm{e}}$, Daniela Gattini ${ }^{\mathrm{f}}$, María Francisca Jaime ${ }^{\mathrm{f}}$, Allan Sharp ${ }^{\mathrm{a}}$
}

\begin{abstract}
aDepartamento de Cirugía Digestiva, División de Cirugía, Facultad de Medicina, Pontificia Universidad Católica de Chile

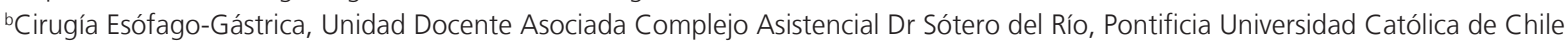
'Residente Cirugía General, Facultad de Medicina, Pontificia Universidad Católica de Chile dInterno de Medicina, Facultad de Medicina, Pontificia Universidad Católica de Chile eSección Cirugía Pediátrica, División de Cirugía, Facultad de Medicina, Pontificia Universidad Católica de Chile fDepartamento de Gastroenterología, Hepatología y Nutrición Pediátrica, División de Pediatría, Facultad de Medicina, Pontificia Universidad Católica de Chile
\end{abstract}

Recibido el 20 de septiembre de 2018; aceptado el 15 de noviembre de 2018

\section{Resumen}

Introducción: La acalasia es el trastorno motor primario más frecuente del esófago. Su incidencia reportada es baja, aún más en pacientes pediátricos. La miotomía de Heller laparoscópica corresponde al estándar actual de tratamiento. Durante los últimos años la miotomía endoscópica por vía oral (POEM) se ha posicionado como una alternativa terapéutica segura y tan efectiva como el Heller para la acalasia esofágica. Objetivo: Describir la técnica de POEM y reportar el primer caso pediátrico en nuestro país. Caso clínico: Paciente de 11 años, previamente sano, que se presentó con disfagia ilógica progresiva y baja de peso. El estudio concluyó una acalasia tipo II. Fue sometido a POEM y cursó un postoperatorio sin incidentes. A un año de la intervención se ha documentado resolución de la sintomatología, seguimiento endoscópico y manométrico sin complicaciones. Conclusiones: El caso descrito corresponde al primer POEM en un paciente pediátrico en nuestro país. La acalasia esofágica es infrecuente en pediatría y el POEM ha demostrado éxito clínico y seguridad comparables a la miotomía de Heller laparoscópica en el corto y mediano plazo. El seguimiento a largo plazo permitirá determinar su rol definitivo en el tratamiento de pacientes pediátricos con acalasia esofágica.
Palabras clave:

Acalasia esofágica; POEM;

miotomía de Heller; endoscopía terapéutica

Correspondencia:

rjmejiam@gmail.com 


\begin{abstract}
Introduction: Achalasia is the most common primary motor disorder of the esophagus. Its reported incidence is low, even more in pediatric patients. Laparoscopic Heller myotomy is the current standard of treatment. During the last years, per-oral endoscopic myotomy (POEM) has been positioned as a safe and effective therapeutic alternative as the Heller procedure for esophageal achalasia. $\mathbf{O b}$ jective: To describe the POEM technique and report the first pediatric case in our country. Clinical Case: 11-year-old patient, previously healthy, who presented with progressive dysphagia for solids and liquids and weight loss. The study concluded a type II achalasia. The patient underwent a POEM and had a postoperative course without incidents. One year after the intervention, symptomatic, endoscopic and manometric resolution have been documented. Conclusions: The described case is the first POEM in a pediatric patient in our country. Esophageal achalasia is uncommon in pediatrics and POEM has demonstrated clinical success and safety comparable to laparoscopic Heller myotomy in short and medium term. Long-term follow-up will determine its definitive role in the treatment of pediatric patients with esophageal achalasia.
\end{abstract}

Keywords:

Esophageal achalasia; POEM;

Heller myotomy; therapeutic endoscopy

\section{Introducción}

La acalasia es el trastorno motor primario más frecuente del esófago. Es una enfermedad rara, caracterizada por ausencia de peristalsis del cuerpo esofágico y relajación incompleta o ausente del esfínter esofágico inferior ${ }^{1}$.

Su incidencia reportada es baja, en pacientes pediátricos se estima anualmente en 0,11 por 100.000 habitantes, sin predilección por raza o género ${ }^{2}$ y la edad promedio al diagnóstico es de 10 años 3 .

Los síntomas más comunes son disfagia, regurgitación, dolor retroesternal y vómitos luego de la alimentación ${ }^{4}$. Puede asociarse a baja de peso, falla de medro ${ }^{4}$ e incluso presentarse de manera atípica; con tos o infecciones pulmonares recurrentes secundarias a aspiración ${ }^{5}$.

En parte por el número limitado de pacientes pediátricos con acalasia, el estándar de tratamiento ha sido controversial ${ }^{3}$. El manejo tradicional incluye la dilatación endoscópica con balón y la miotomía de Heller laparoscópica asociada o no a fundoplicatura parcial ${ }^{6}$. La inyección de toxina botulínica, uso de bloqueadores de los canales de calcio o nitratos de larga acción, son terapias menos efectivas en el largo plazo y probablemente son consideradas como puente a la cirugía o paliación en quienes está contraindicado el manejo quirúrgico definitivo ${ }^{7}$.

El 2007 Pasricha describió la técnica de miotomía endoscópica en un modelo porcino ${ }^{8}$, y el 2010 Inoue reportó los primeros resultados en humanos ${ }^{9}$. Desde entonces, el POEM (por las siglas en inglés per-oral endoscopic myotomy) se ha instalado como una nueva alternativa terapéutica en el manejo de la acalasia ${ }^{7}$. La experiencia acumulada en adultos ha demostrado resultados que lo posicionan como una técnica segura y tan efectiva como la miotomía de Heller. El procedi- miento se ha introducido gradualmente en población pediátrica, existiendo reportes de resultados iniciales?

El objetivo del presente manuscrito es comunicar el primer caso, en nuestro país, de un paciente pediátrico con acalasia sometido a POEM y su seguimiento a la fecha.

\section{Caso clínico}

Paciente de género masculino de 11 años de edad, con antecedente de rinitis alérgica y dermatitis atópica, eutrófico y con buen desarrollo psicomotor. Consultó de forma ambulatoria por cuadro de dos meses de evolución de disfagia ilógica progresiva, sin vómitos, pirosis ni regurgitación. Destacaba baja de peso de 4 $\mathrm{kg}$ en los últimos cuatro meses, sin dolor abdominal.

Dentro del estudio diagnóstico se realizó una endoscopía digestiva alta que resultó dentro de límites normales y biopsia esofágica informada sin alteraciones.

Fue solicitado un estudio esofágico contrastado, que identificó hallazgos compatibles con acalasia esofágica (figura 1). Se complementó con una manometría esofágica de alta resolución, cuyo informe describió una mediana de presión de relajación integrada de $36 \mathrm{mmHg}$ ( $\mathrm{VN}<15 \mathrm{mmHg}$ ), fenómenos de panpresurización esofágica después de las degluciones múltiples y aperistalsis del cuerpo esofágico, concordante con un patrón de acalasia tipo II según la clasificación de Chicago modificada (figura 2).

Confirmado el diagnóstico se discutió el caso en equipo multidisciplinario y se decidió, previo acuerdo con sus padres, resolución por vía endoscópica mediante POEM. El puntaje de Eckardt ${ }^{10}$ preoperatorio era de 10 puntos (disfagia 3 puntos, dolor retroesternal 3 puntos, regurgitación 3 puntos y baja de peso 1 punto). 


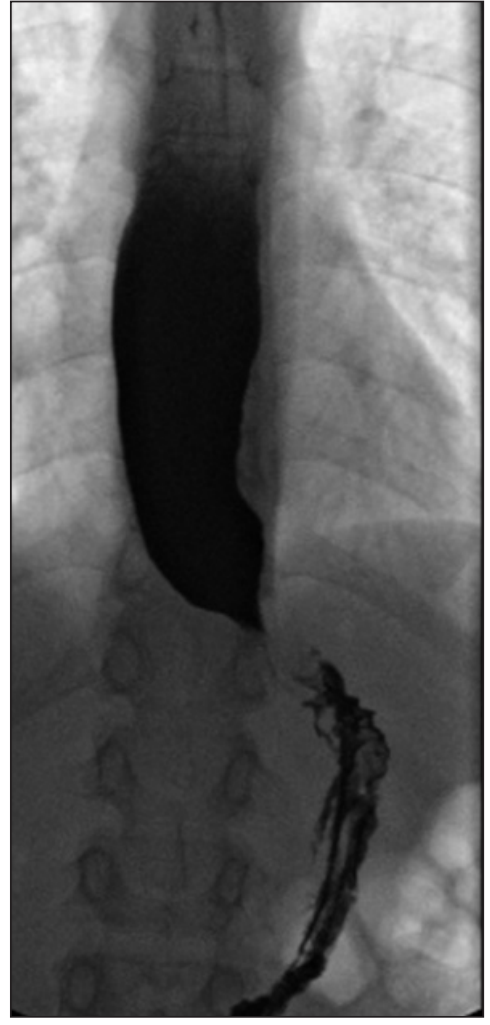

Figura 1. Estudio esofágico contrastado preoperatorio. Se evidencia a nivel de la unión gastroesofágica una estenosis en segmento corto, con la morfología característica en pico de pájaro, dilatación del esófago en su tercio medio y paso filiforme del medio de contraste a la cámara gástrica.
El procedimiento se llevó a cabo en pabellón, bajo anestesia general y en decúbito supino. Se utilizó un endoscopio diagnóstico con espaciador distal oblicuo, insuflador de $\mathrm{CO}_{2}$ y equipo de electrocirugía $\mathrm{ERBE}^{\oplus}$. La endoscopía inicial mostró contenido alimentario en esófago, que se aspiró previamente a iniciar la intervención. La unión gastroesofágica (UGE) fue medida a 38 centímetros de la arcada dentaria.

Se realizó la elevación de la mucosa con una solución de Voluven e éndigo carmín y luego una mucotomía longitudinal entre las 2 y las 3 de las manecillas del reloj en la cara anterior del esófago (figuras $3 \mathrm{~A}$ y 3B), aproximadamente 11 centímetros proximales a la UGE. Posteriormente se procedió a la creación de un túnel submucoso (figura 3C), mediante técnica de disección submucosa y con cuchillo endoscópico Flush Knife $\mathrm{BT}^{\circledast} 2,5 \mathrm{~mm}$, que se extendió hasta los 41 centímetros de la arcada dentaria. La extensión distal a la UGE fue comprobada a través de retrovisión endoscópica en la cámara gástrica para objetivar la tinción de la de la submucosa subcardial, sumado a la medición endoscópica, identificación de las fibras musculares oblicuas del estómago y los vasos de la submucosa gástrica.

La miotomía de las fibras circulares internas de la muscular esofágica fue realizada con cuchillo endoscópico de punta triangular (TTKnife ${ }^{\varpi}$ ), desde los 30 a los 41 centímetros de la arcada (figura 3D). Posteriormente se aseguró la hemostasia e instiló el túnel con una solución de gentamicina. La mucotomía fue cerrada

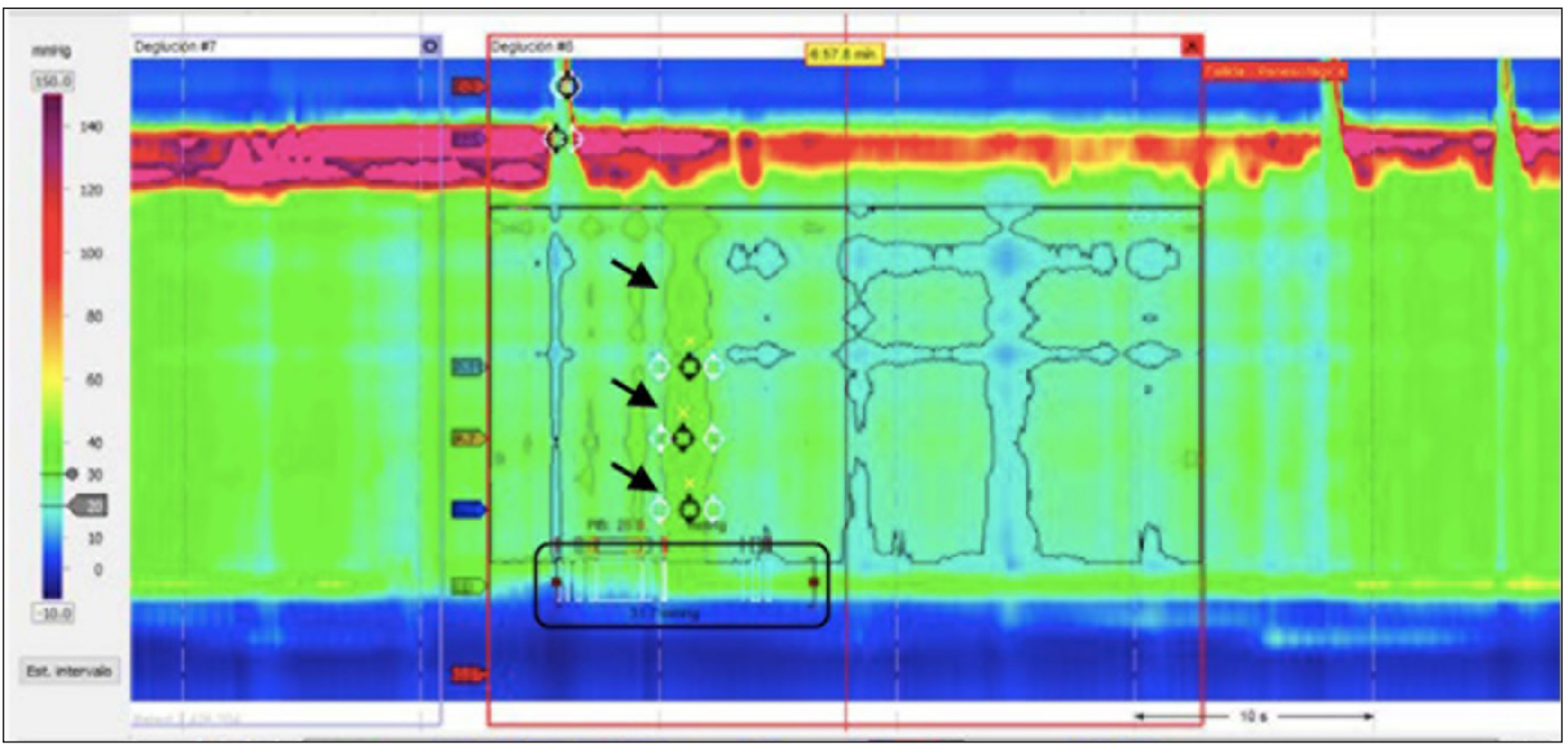

Figura 2. Manometría esofágica de alta resolución. En degluciones protocolizadas es evidente la ausencia de relajación de la unión gastroesofágica (área demarcada) y fenómenos de panpresurización (flechas). 

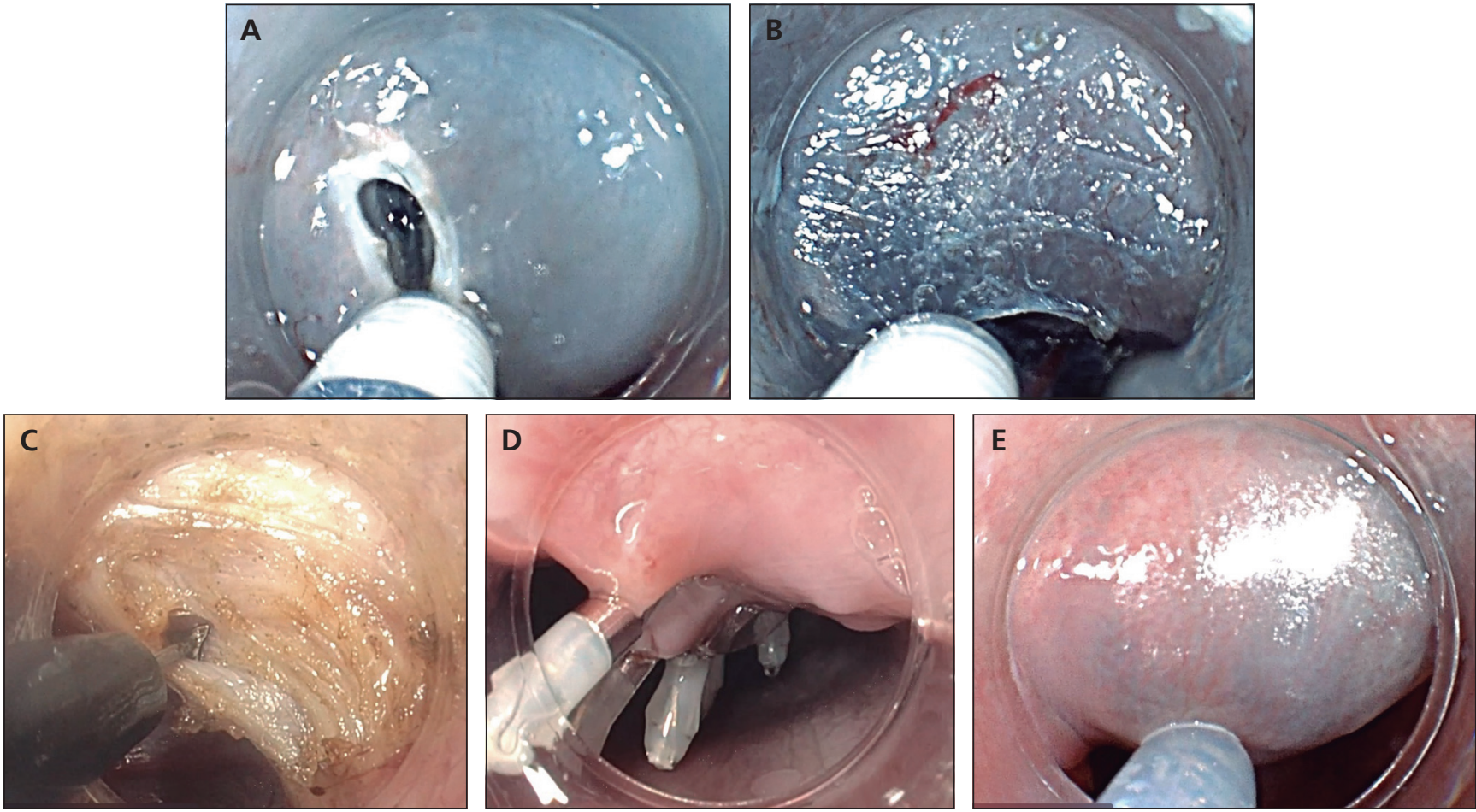

Figura 3. Secuencia de pasos en la técnica POEM. (A) Elevación de la mucosa. (B) Mucotomía longitudinal. (C) Creación del túnel submucoso. (D) Miotomía de las fibras circulares internas del esófago. (E) Cierre de la mucotomía con clips.

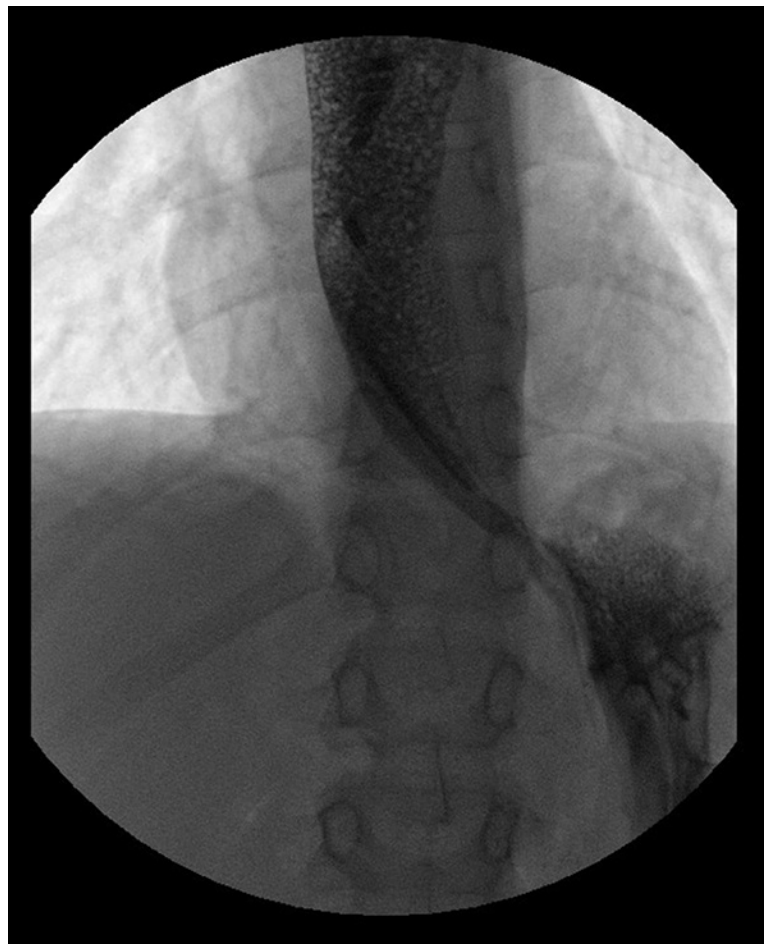

Figura 4. Estudio esofágico contrastado postoperatorio. Se observan clips en el tercio distal del esófago, con paso de contraste al estómago. Ya no es visible la zona de obstrucción previamente descrita en la unión gastroesofágica y no se identifican trayectos extraluminales de contraste sugerentes de filtración. con clips endoscópicos (figura 3E) y en la etapa final se aseguró el paso fácil del instrumento por la UGE.

El paciente se mantuvo en régimen cero hasta la realización de un estudio esofágico con contraste hidrosoluble, dentro de las primeras $24 \mathrm{~h}$, que descartó filtración (figura 4). Posteriormente fue alimentado con régimen líquido, con buena tolerancia, siendo dado de alta al segundo día, con indicación de inhibidor de bomba de protones (IBP) en dos dosis diarias.

Fue controlado una semana luego de la intervención, objetivando resolución de los síntomas y buena tolerancia al régimen. Se progresó a papilla y se indicó incorporar progresivamente otros alimentos.

A los tres meses postoperatorio se controló con una endoscopía digestiva alta, que demostró buen paso del instrumento a través de la UGE, ausencia de elementos de mal vaciamiento esofágico o evidencias de reflujo gastroesofágico (RGE). La manometría esofágica de alta resolución demostró ausencia de fenómenos de presurización esofágica y una mediana de presión de relajación integrada de $15 \mathrm{mmHg}$. El paciente se mantiene asintomático, sin clínica de RGE ni requerimiento de IBP. La endoscopía digestiva alta realizada al año no presenta cambios respecto a la previa. Su puntaje de Eckardt al seguimiento es de uno (disfagia ocasional). 


\section{Discusión}

La acalasia esofágica es un diagnóstico poco común en pediatría ${ }^{11}$. Los tratamientos disponibles incluyen alternativas farmacológicas, endoscópicas y quirúrgicas, siendo las más comúnmente usadas la dilatación endoscópica con balón y la miotomía de Heller laparoscópica. Entre estas dos últimas se reconoce la superioridad de la miotomía de Heller por relacionarse con menores tasas de recurrencia que la dilatación con balón ${ }^{2}$. Actualmente el manejo óptimo en pacientes pediátricos no ha sido definido y la literatura aún carece de protocolos estandarizados de seguimiento ${ }^{4}$.

El POEM ha surgido como una alternativa terapéutica competitiva en el tratamiento de la acalasia durante la última década, porque combina los beneficios de un procedimiento endoscópico y por lo tanto mínimamente invasivo, con la eficacia de una miotomía quirúrgica ${ }^{2}$. Algunos autores consideran a su favor que evita la disección excesiva del hiato esofágico, las cicatrices y permite reintegración a la actividad en pocos días ${ }^{12,13}$, además de la planificación del largo de la miotomía de acuerdo al estudio manométrico y endoscópico ${ }^{2}$. Los estudios de seguimiento disponibles reportan tasas de éxito clínico (Eckardt $<3)$ mayores a un $90 \%$ y mejoría en el perfil de presiones de la manometría esofágica ${ }^{13-15}$. Durante los últimos años se han publicado series en pacientes pediátricos, con buenos resultados en la resolución de la sintomatología, registrándose incluso hasta $100 \%$ a 24,6 meses del procedimiento, sin complicaciones perioperatorias graves (Clavien > III) ni en el seguimiento a mediano plazo $^{11}$.

La técnica tiene un buen perfil de seguridad; el capnoperitoneo y el enfisema submucoso son consecuencias inevitables y pueden ser fácilmente manejados intra procedimiento si son clínicamente significativos ${ }^{9,16}$.

Los datos disponibles aún no son suficientes para establecer recomendaciones en la aplicación de la técnica según límites de peso y edad ${ }^{2}$. El paciente más joven reportado corresponde al caso publicado por Maselli et al: paciente de tres años de edad, portador de trisomía 21 y desnutrición severa secundaria a acalasia esofágica tratado mediante POEM con éxito ${ }^{17}$.

Es importante reconocer la curva de aprendizaje asociada a la ejecución del procedimiento, que ha sido establecida cerca de los 20 casos $^{18}$; los mejores resultados se obtienen en centros con experiencia en endoscopía terapéutica ${ }^{11}$.
Actualmente se considera que todos los pacientes con acalasia pueden ser tratados mediante POEM, incluso se ha establecido como alternativa terapéutica en otros trastornos motores del esófago como el espasmo esofágico difuso, el esófago en cascanueces y el esófago hipercontráctil (jackhammer) ${ }^{11}$. Sería el tratamiento de elección en la acalasia tipo III porque permite miotomías de mayor longitud y en pacientes con recidiva sintomática post miotomía de Heller o después de un primer POEM, pues provee la alternativa de variar el abordaje evitando zonas de fibrosis².

Si bien actualmente la miotomía de Heller laparoscópica es el tratamiento de elección en niños, el POEM es cada vez más utilizado como alternativa terapéutica. Cuenta con resultados prometedores del punto de vista de efectividad y seguridad en el entorno pediátrico, y el presente reporte constituiría, a nuestro conocimiento, el primer caso realizado en el país. Los resultados de la técnica deben ser respaldados en estudios multicéntricos de mayor volumen y con seguimiento en el largo plazo, para constituirlo como la alternativa de elección en esta población.

\section{Responsabilidades éticas}

Protección de personas y animales: Los autores declaran que los procedimientos seguidos se conformaron a las normas éticas del comité de experimentación humana responsable y de acuerdo con la Asociación Médica Mundial y la Declaración de Helsinki.

Confidencialidad de los datos: Los autores declaran que han seguido los protocolos de su centro de trabajo sobre la publicación de datos de pacientes.

Derecho a la privacidad y consentimiento informado: Los autores han obtenido el consentimiento informado de los pacientes y/o sujetos referidos en el artículo. Este documento obra en poder del autor de correspondencia.

\section{Conflicto de intereses}

Los autores declaran no tener conflicto de intereses. 


\section{Referencias}

1. Li C, Tan Y, Wang X, Liu D. Peroral endoscopic miotomy for treatment of achalasia in children and adolescents. J Ped Surg 2015; 50: 201-5.

2. Caldaro T, Familiari P, Romeo E, et al. Treatment of esophageal achalasia in children: today and tomorrow. J Ped Surg 2015; 50: 726-30.

3. Marlais M, Fishman JR, Fell JME, et al. UK incidence of achalasia: an 11-year national epidemiological study. Arch Dis Child 2011; 96, 192-4.

4. Chen W, Li Q, Zhou P, et al. Long-term outcomes of peroral endoscopic myotomy for achalasia in pediatric patients: a prospective, single-center study. Gastrointest Endosc 2015; 81 (1): 91-100.

5. Franklin A, Petrosyan M, Kane T. Childhood achalasia: A comprehensive review of disease, diagnosis and therapeutic management. World J Gastrointest Endosc 2014; 6 (4): 105-11.

6. Vaezi MF, Pandolfino JE, Vela MF. ACG clinical guideline: diagnosis and Management of achalasia. Am J Gastroenterol 2013; 108: 1238-49.

7. Kethman W, Thorson C, Sinclair T, et al. Initial experience with peroral endoscopic myotomy for treatment of achalasia in children. J Pediatr Surg. 2018;53(8):15326.

8. Pasricha P, Hawari R, Ahmed I, et al. Submucosal endoscopic esophageal myotomy: a novel experimental approach for the treatmen of achalasia. Endoscopy 2007;39:761-4

9. Inoue $\mathrm{H}$, Minami $\mathrm{H}$, Kobayashi $\mathrm{Y}$, et al. Peroral endoscopic myotomy (POEM) for esophageal achalasia. Endoscopy 2010;42: 265-71.

10. Eckardt A, Eckardt V. Treatment and Surveillance strategies in achalasia: an update. Nat Rev Gastroenterol. Hepatol 2011;8:311-9.

11. Li Q, Zhou P. Perspective on peroral endoscopic myotomy for achalasia: Zhongshan experience. Gut and Liver 2015;9(2):152-8.

12. Simic AP, Radovanovic NS, Skrobic $\mathrm{OM}$, et al. Significance of limited hiatal dissection in surgery for achalasia. J Gastrointest Surg 2010;14:587-93.

13. Ramchandani M, Reddy DN, Darisetty S. Peroral endoscopic myotomy for achalasia cardia: Treatment analysis and follow up of over 200 consecutive patients at a single center. Digest Endosc 2016;28:19-26.
14. Familiari P, Gigante G, Marchese M, et al. Peroral endoscopic myotomy for esophageal achalasia: outcomes of the first 100 patients with short term follow up. Ann Surg 2016;263(1):82-7.

15. Talukdar R, Inoue $H$, Nageshwar Reddy D. Efficacy of peroral endoscopic myotomy POEM in the treatment of achalasia: a systematic review and metaanalysis. Surg Endosc 2015;29(11):303046.

16. Li QL, Chen WF, Zhou PH, et al. Peroral endoscopic myotomy for the treatment of achalasia: a clinical comparative study of endoscopic ful thickness and circular muscle myotomy. J Am Coll Surg 2013;217:442-51.

17. Maselli R, Inoue $\mathrm{H}$, Misawa $\mathrm{M}$, et al. Per oral endoscopic myotomy (POEM) in a 3 years old girl with severe growth retardation, achalasia and Down syndrome. Endoscopy 2012; 44: E285-7.

18. Sharata AM, Dunst CM, Pescarus R, Shlomovitz E, Willie AJ, Reavis KM, Swanstrom L. Peroral endoscopic myotomy (POEM) for esophageal primary motility disorders: analysis of 100 consecutive patients. J Gastrointest Surg 2015;19(1):161-70. 